\title{
Light Scattering from a Random Assembly of Anisotropic Plates in Two- and Three-Dimensional Space*
}

\author{
Masaru Matsuo, Shunji Nomura**, Takeji Hashimoto, \\ and Hiromichi KAWAI \\ Department of Polymer Chemistry, Faculty of Engineering, \\ Kyoto University, Kyoto, Japan. \\ (Received September 25, 1973)
}

\begin{abstract}
Light-scattering patterns are calculated for a random assembly of anisotropic plates having finite dimensions. The results are compared with those calculated previously for a random assembly of one-dimensional rods having infinitesimally small lateral dimensions, and with those measured for the denatured collagen films. The effect of the finite lateral dimensions on the rod-like scattering is shown to be important in accounts for the experimental scattered intensity distributions at large scattering angles.

KEY WORDS Light-Scattering / Nonspherulitic Scattering / Rod-

Like Textures / Anisotropic Plates / Random Assembly / Denatured Collagen Films /
\end{abstract}

In the previous papers, the light scattering from nonspherulitic or prespherulitic crystalline superstructures having fibrillar or sheet-like appearances under observation of light and electron microscopes was explained in terms of the isolated rod theories. ${ }^{1-6}$

The scattering labelled as rod-like were found for crystalline polymer films such as poly(tetrafluoroethylene), ${ }^{7,9}$ poly(chlorotrifluoroethylene), ${ }^{8-10}$ collagen, ${ }^{2,5,6,11}$ cellulose derivatives ${ }^{12,13}$ and etc. Theories of rod-like scattering were developed for various models; one-dimensional homogeneous rods with infinitesimally small lateral size oriented randomly in (i) two-dimensional space, ${ }^{1,6}$ or (ii) three-dimensional space, ${ }^{2,5}$ (iii) homogeneous cylindrical and disklike particles $^{3,4}$ oriented randomly in three-dimensional space. These types of scattering turned out to be qualitatively identical.

In one of the previous papers, the one-dimensional rod theory was quantitatively tested by comparing the theoretical and experimental

* Presented partly at the 21st Symposium on Polymer Chemistry, Osaka, Japan, November 2, 1972.

** Present address: Division of Macromolecular Science, Case Western Reserve University, Cleveland, Ohio, U.S.A.

+ To whom correspondence should be addressed. intensity distributions. ${ }^{6}$ Deviations from the theory were observed at small scattering angles where the interparticle interference effect is significant and at large scattering angles. The deviations at large scattering angles were partly accounted for by taking account of the optical inhomogeneities of the rods associated with fine structure of the texture. ${ }^{6}$

In this paper we shall deal with the scattering from a random assembly of anisotropic plates with finite dimensions partly to generalize the model of the rod-like texture and partly to search for a hypothesis that would account for the deviations at large scattering angles. ${ }^{6}$

\section{THE MODEL AND GENERAL CALCULATIONS}

We shall calculate first $H_{\mathrm{v}}$ and $V_{\mathrm{v}}$ scattering (horizontal and vertical components of scattered light observed by using a vertically polarized incident beam, respectively) from the anisotropic plates with given orientation specified by angles $\Theta, \phi$, and $\Phi$ or angles $\alpha, \beta$, and $\gamma$. In Figures 1 and 2 are shown the model and the Cartesian coordinate systems.

The incident beam (whose propagation direction is denoted by a unit vector $s_{0}$ ) enters into the assembly along the $O X$ axis, and the 


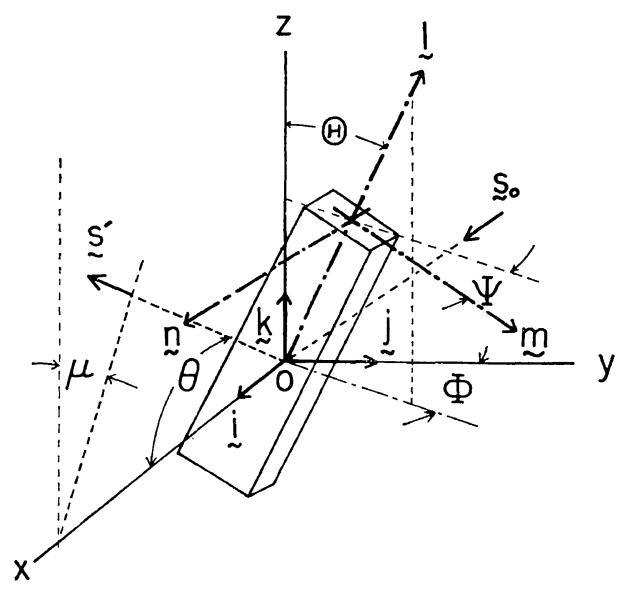

Figure 1. The coordinate systems used in this study. The vectors $i, j$, and $\boldsymbol{k}$ are the unit vectors along the coordinate system fixed to the apparatus, and $\boldsymbol{l}, \boldsymbol{m}$, and $\boldsymbol{n}$ are those fixed to the anisotropic plate.

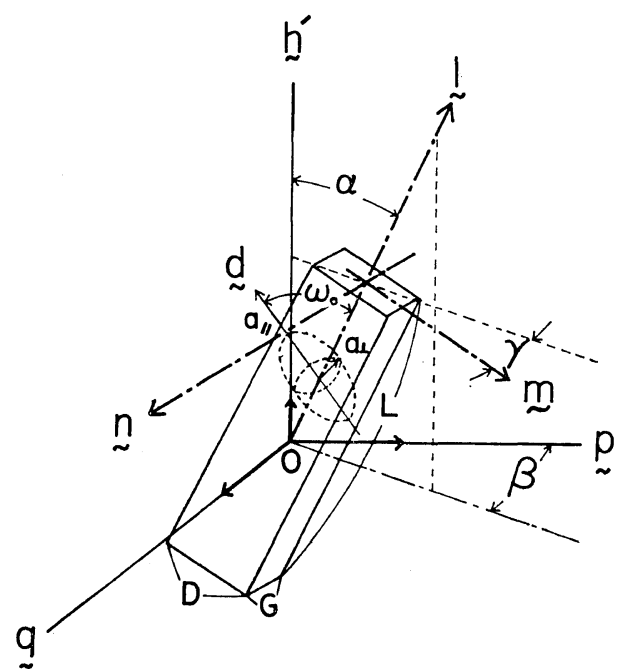

Figure 2. The model of anisotropic plate. The plate has dimensions of $L, D$, and $G$ along the vectors $\boldsymbol{l}, \boldsymbol{m}$, and $\boldsymbol{n}$. The optical axis of the uniaxially anisotropic scattering element is specified by a unit vector $\boldsymbol{d}$, and makes an angle $\omega_{0}$ with respect to $\boldsymbol{l}$ and is parallel to the plane of $\boldsymbol{l}$ and $\boldsymbol{m}$. The vector $\boldsymbol{h}^{\prime}$ is a unit vector along the scattering vector $s$.

scattered beam (whose direction is denoted as a unit vector $\boldsymbol{s}^{\prime}$ ) is detected as a function of $\theta$, scattering angle and $\mu$, azimuthal angle taken from the vertical direction, $O Z$. The vectors $i$, $\boldsymbol{j}$, and $\boldsymbol{k}$ are the unit vectors along the coordinate axes (Figure 1). The vectors $\boldsymbol{l}, \boldsymbol{m}$, and $n$ are the unit vectors along three principal axes of the plate. The plates are randomly oriented with respect to the angles $\Theta, \psi$, and $\Phi$ in threedimensional space or with respect to the angle $\Theta$ under a condition of $\Phi=0^{\circ}$ in two-dimensional space.

The plate is assumed to be composed of uniaxially anisotropic scattering elements with polarizabilities $\alpha_{\| /}$and $\alpha_{\perp}$ along and perpendicular to the principal optical axis whose direction is defined by a unit vector $d$, respectively (Figure 2). As shown in Figure 2, the optical axes are assumed to orient parallel to the plane composed of the vectors $\boldsymbol{l}$ and $\boldsymbol{m}$ with an angle $\omega_{0}$ with respect to the vector $l$. The plate has dimensions of $L, D$, and $G$ along the vectors $l, m$, and $n$, respectively.

The amplitude of the scattered light from the plate having a given orientation with respect to the reference coordinate is given, according to the Rayleigh-Gans theory, ${ }^{14}$ by

$$
\begin{aligned}
E= & C \int_{r_{1}=-L / 2}^{L / 2} \int_{r_{2}=-D / 2}^{D / 2} \int_{r_{3}=-G / 2}^{G / 2}(\boldsymbol{M} \cdot \boldsymbol{O}) \\
& \times \exp [i(\boldsymbol{h} \cdot \boldsymbol{r})] \mathrm{d} r_{1} \mathrm{~d} r_{2} \mathrm{~d} r_{3}
\end{aligned}
$$

where $r$ is a vector within the plate and is given by

$$
\boldsymbol{r}=r_{1} \boldsymbol{l}+r_{2} \boldsymbol{m}+r_{3} \boldsymbol{n}
$$

The vector $\boldsymbol{h}$ is defined by $(2 \pi / \lambda) s$, where the vector $s$ is the scattering vector defined by $\left(s_{0}-s^{\prime}\right)$. Therefore

$$
\begin{aligned}
\boldsymbol{h}= & (2 \pi / \lambda)[(1-\cos \theta) \boldsymbol{i}-(\sin \theta \sin \mu) \boldsymbol{j} \\
& -(\sin \theta \cos \mu) \boldsymbol{k}] \\
= & (4 \pi / \lambda) \sin (\theta / 2) \cdot \boldsymbol{h}^{\prime}
\end{aligned}
$$

where $\boldsymbol{h}^{\prime}$ is a unit vector defined by

$$
\boldsymbol{h}^{\prime}=\boldsymbol{i} \sin (\theta / 2)-\boldsymbol{j} \cos (\theta / 2) \sin \mu-\boldsymbol{k} \cos (\theta / 2) \cos \mu
$$

The vector $\boldsymbol{M}$ is associated with the induced dipole moment of the scattering element located at $\boldsymbol{r}$ from the center of the plate, and is given, for a vertically polarized incident beam, by

$$
\boldsymbol{M}=E_{0}\left\{\delta_{0}(\boldsymbol{k} \cdot \boldsymbol{d}) \boldsymbol{d}+b_{\mathrm{t}} \boldsymbol{d}\right\}
$$

where $E_{0}$ is the field strength of the incident beam. The quantity $\delta_{0}$ is the anisotropy of the 
Light Scattering from Plate-like Crystalline Textures

scattering element defined as $\left(\alpha_{\|}-\alpha_{\perp}\right)$, and $b_{\mathrm{t}}$ is defined as $\left(\alpha_{\perp}-\alpha_{\mathrm{s}}\right)$ where $\alpha_{\mathrm{s}}$ is polarizability of the surrounding medium of the plates. The vector $\boldsymbol{d}$ is given by

$$
\boldsymbol{d}=\boldsymbol{l} \cos \omega_{0}+\boldsymbol{m} \sin \omega_{0}
$$

The vector $\boldsymbol{O}$ is a unit vector along the polarization direction of the analyzer set in between the specimen and the detector registering the scattering. At small scattering angles, the vector $\boldsymbol{O}$ is approximated by the vectors $\boldsymbol{j}$ and $\boldsymbol{k}$ for $H_{\checkmark}$ and $V_{\checkmark}$ polarizations, respectively. ${ }^{15,16}$

If the plate and its surrounding medium are homogeneous, as we assume in this report, the quantities $\delta_{0}, b_{t}$, and $\omega_{0}$ are constant within the plate, so that the quantity $(\boldsymbol{M} \cdot \boldsymbol{O})$ is put outside the integral of eq 1 . Therefore the eq 1 can be evaluated by giving the relationship between the coordinated systems, $(\boldsymbol{i}, \boldsymbol{j}, \boldsymbol{k})$ and $(\boldsymbol{l}, \boldsymbol{m}, \boldsymbol{n})$.

\section{RANDOM ASSEMBLY OF THE ANISOTROPIC PLATES IN THREE-DIMENSIONAL SPACE}

When the plates are randomly oriented in three-dimensional space, it is easier to calculate the scattering in terms of the coordinate system shown in Figure 2 rather than to calculate in terms of the coordinate system shown in Figure 1. In Figure 2, the vector $\boldsymbol{h}^{\prime}$ is a unit vector along the vector $s$ and is given by eq 4 . The unit vector $\boldsymbol{p}$ is set perpendicular to the vector $\boldsymbol{h}^{\prime}$ in the plane composed of the vectors $\boldsymbol{h}^{\prime}$ and $\boldsymbol{k}^{\prime}$ and the unit vector $\boldsymbol{q}$ is set perpendicular to both $\boldsymbol{h}^{\prime}$ and $\boldsymbol{p}$ in a direction given by $\boldsymbol{p} \times \boldsymbol{h}^{\prime}$.

The orthogonal matrices of the coordinate transformation are given by

$$
\left(\begin{array}{l}
\boldsymbol{l} \\
\boldsymbol{m} \\
\boldsymbol{n}
\end{array}\right)=\left(\begin{array}{ccc}
\cos \alpha & \sin \alpha \sin \beta & \sin \alpha \cos \beta \\
-\sin \alpha \cos \gamma & \cos \alpha \sin \beta \cos \gamma+\cos \beta \sin \gamma & \cos \alpha \sin \beta \cos \gamma-\sin \beta \sin \gamma \\
\sin \alpha \sin \gamma & -\cos \alpha \sin \beta \sin \gamma+\cos \beta \cos \gamma & -\cos \alpha \cos \beta \sin \gamma-\sin \beta \cos \gamma
\end{array}\right)\left(\begin{array}{l}
\boldsymbol{h}^{\prime} \\
\boldsymbol{q} \\
\boldsymbol{p}
\end{array}\right)
$$

and

$$
\left(\begin{array}{l}
\boldsymbol{h}^{\prime} \\
\boldsymbol{p} \\
\boldsymbol{q}
\end{array}\right)=\left(\begin{array}{lll}
\sin (\theta / 2) & -\cos (\theta / 2) \sin \mu & -\cos (\theta / 2) \cos \mu \\
\boldsymbol{J}^{-1 / 2} \sin (\theta / 2) \cos (\theta / 2) \cos \mu & -J^{-1 / 2} \cos ^{2}(\theta / 2) \sin \mu \cos \mu & J^{1 / 2} \\
\boldsymbol{J}^{-1 / 2} \cos (\theta / 2) \sin \mu & J^{-1 / 2} \sin (\theta / 2) & 0
\end{array}\right)\left(\begin{array}{l}
\boldsymbol{i} \\
\boldsymbol{j} \\
\boldsymbol{k}
\end{array}\right)
$$

where $J=1-\cos ^{2}(\theta / 2) \cos ^{2} \mu$.

From eq 1 to 3 and 7 , it follows that

$$
\begin{aligned}
E & =C(\boldsymbol{M} \cdot \boldsymbol{O}) \int_{-L / 2}^{L / 2} \mathrm{e}^{i(h \cdot l) r_{1}} \mathrm{~d} r_{1} \int_{-D / 2}^{D / 2} \mathrm{e}^{i(h \cdot m) r_{2}} \mathrm{~d} r_{2} \int_{-G / 2}^{G / 2} \mathrm{e}^{i(h \cdot n) r_{3}} \mathrm{~d} r_{3} \\
& =C(\boldsymbol{M} \cdot \boldsymbol{O}) V \frac{\sin \left[U_{1} \cos \alpha\right]}{\left[U_{1} \cos \alpha\right]} \frac{\sin \left[U_{\mathrm{d}} \sin \alpha \cos \gamma\right]}{\left[U_{\mathrm{d}} \sin \alpha \cos \gamma\right]} \frac{\sin \left[U_{\mathrm{g}} \sin \alpha \sin \gamma\right]}{\left[U_{\mathrm{g}} \sin \alpha \sin \gamma\right]}
\end{aligned}
$$

where $V=L D G$, volume of the plate, and $U_{1}, U_{\mathrm{d}}$, and $U_{\mathrm{g}}$ are defined by,

$$
U_{1}=(2 \pi L / \lambda) \sin (\theta / 2), \quad U_{\mathrm{d}}=(2 \pi D / \lambda) \sin (\theta / 2), \quad U_{\mathrm{g}}=(2 \pi G / \lambda) \sin (\theta / 2)
$$

The effective induced dipole moment $(\boldsymbol{M} \cdot \boldsymbol{O})$ in eq 9 is given from eq 5 to 8 , by

$$
\begin{aligned}
(\boldsymbol{M} \cdot \boldsymbol{O})_{H_{\mathrm{V}}}= & \delta_{0}\left\{\left(\sin \omega_{0} \sin \alpha \cos \gamma-\cos \omega_{0} \cos \alpha\right) \cos (\theta / 2) \cos \mu\right. \\
& \left.+\left(\cos \omega_{0} \sin \alpha \cos \beta+\sin \omega_{0} \cos \alpha \cos \beta \cos \gamma-\sin \omega_{0} \sin \beta \sin \gamma\right) J^{1 / 2}\right\} \\
& \times\left\{\left(\cos \omega_{0} \sin \alpha \sin \beta+\sin \omega_{0} \cos \alpha \sin \beta \cos \gamma+\sin \omega_{0} \cos \beta \sin \gamma\right) \sin (\theta / 2) J^{-1 / 2}\right. \\
& -\left(\sin \omega_{0} \cos \alpha \cos \beta \cos \gamma+\cos \omega_{0} \sin \alpha \cos \beta-\sin \omega_{0} \sin \beta \sin \gamma\right) \\
& \left.\times \cos ^{2}(\theta / 2) \sin \mu \cos \mu J^{-1 / 2}+\left(\sin \omega_{0} \sin \alpha \cos \gamma-\cos \omega_{0} \cos \alpha\right) \cos (\theta / 2) \sin \mu\right\}
\end{aligned}
$$

for the $H_{\checkmark}$ scattering, and

$$
\begin{aligned}
(\boldsymbol{M} \cdot \boldsymbol{O})_{V_{\mathrm{v}}=} & \delta_{0}\left\{\left(\sin \omega_{0} \sin \alpha \cos \gamma-\cos \omega_{0} \cos \alpha\right) \cos (\theta / 2) \cos \mu+\left(\cos \omega_{0} \sin \alpha \cos \beta\right.\right. \\
& \left.\left.+\sin \omega_{0} \cos \alpha \cos \beta \cos \gamma-\sin \omega_{0} \sin \beta \sin \gamma\right) J^{1 / 2}\right\}^{2}+b_{\mathrm{t}}
\end{aligned}
$$


M. Matsuo, S. Nomura, T. Hashimoto, and H. KawaI

for the $V_{\nabla}$ scattering.

Average $H_{\nabla}$ and $V_{\nabla}$ scattered intensities from the assembly are given, by neglecting the interplate interference of the scattered waves, by

$$
I=\frac{1}{8 \pi^{2}} \int_{\alpha=0}^{\pi} \int_{\beta=0}^{2 \pi} \int_{\gamma=0}^{2 \pi} E^{2} \sin \alpha \mathrm{d} \alpha \mathrm{d} \beta \mathrm{d} \gamma
$$

Therefore $H_{\mathrm{v}}$ and $V_{\nabla}$ scattered intensities can be calculated from eq 9 to 13 , and are given by

$$
\begin{aligned}
& I_{H_{\mathrm{V}}}=K_{1}\left\{I_{1}(\theta) \sin ^{2} 2 \mu+I_{2}(\theta)\right\} \\
& I_{V_{\mathrm{V}}}=K_{2}\left\{I_{3}(\theta) \cos 4 \mu+I_{4}(\theta) \cos 2 \mu+I_{5}(\theta)\right\}
\end{aligned}
$$

where the $I_{i}$ terms are given by

$$
\begin{aligned}
& I_{1}(\theta)=\left[35\left\{H_{33}(\theta)-2 H_{23}(\theta)+H_{13}(\theta)+6 H_{32}(\theta)-6 H_{22}(\theta)+H_{31}(\theta)\right\} \cos ^{4} \omega_{0}\right. \\
& +10\left\{-7 H_{33}(\theta)+14 H_{23}(\theta)-7 H_{13}(\theta)-21 H_{32}(\theta)+18 H_{22}(\theta)+3 H_{12}(\theta)-3 H_{21}(\theta)\right\} \cos ^{2} \omega_{0} \\
& \left.+35 H_{33}(\theta)-70 H_{23}(\theta)+35 H_{13}(\theta)+30 H_{22}(\theta)-30 H_{12}(\theta)+3 H_{11}(\theta)\right] \cos ^{4}(\theta / 2) \\
& I_{2}(\theta)=I_{21}(\theta) \cos ^{2}(\theta / 2)+I_{22}(\theta) \\
& I_{21}(\theta)=20\left\{-H_{33}(\theta)+2 H_{23}(\theta)-H_{13}(\theta)-6 H_{32}(\theta)+6 H_{22}(\theta)-H_{31}(\theta)\right\} \cos ^{4} \omega_{0} \\
& +8\left\{5 H_{33}(\theta)-10 H_{23}(\theta)+5 H_{13}(\theta)+15 H_{32}(\theta)-12 H_{22}(\theta)-3 H_{12}(\theta)+3 H_{21}(\theta)\right\} \cos ^{2} \omega_{0} \\
& +4\left\{-5 H_{33}(\theta)+10 H_{23}(\theta)-5 H_{13}(\theta)-6 H_{22}(\theta)+6 H_{12}(\theta)-H_{11}(\theta)\right\} \\
& I_{22}(\theta)=\left\{H_{33}(\theta)-2 H_{23}(\theta)+H_{13}(\theta)+6 H_{32}(\theta)-8 H_{22}(\theta)+H_{31}(\theta)\right\} \cos ^{4} \omega_{0} \\
& +2\left\{-H_{33}(\theta)+2 H_{23}(\theta)-H_{13}(\theta)-3 H_{32}(\theta)+2 H_{22}(\theta)+H_{12}(\theta)-H_{21}(\theta)\right\} \cos ^{2} \omega_{0} \\
& +H_{33}(\theta)-2 H_{23}(\theta)+H_{13}(\theta)+2 H_{22}(\theta)-2 H_{12}(\theta)+H_{11}(\theta) \\
& I_{3}(\theta)=\left[\left\{35 H_{33}(\theta)-70 H_{23}(\theta)+35 H_{13}(\theta)+210 H_{32}(\theta)-186 H_{22}(\theta)+35 H_{31}(\theta)\right\} \cos ^{4} \omega_{0}\right. \\
& +2\left\{-35 H_{33}(\theta)+70 H_{23}(\theta)-35 H_{13}(\theta)-105 H_{32}(\theta)-66 H_{22}(\theta)+15 H_{12}(\theta)\right. \\
& \left.-15 H_{21}(\theta)\right\} \cos ^{2} \omega_{0}+35 H_{33}(\theta)-70 H_{23}(\theta)+35 H_{13}(\theta)+54 H_{22}(\theta) \\
& \left.-30 H_{12}(\theta)+3 H_{11}(\theta)\right] \cos ^{4}(\theta / 2) \\
& I_{4}(\theta)=4 I_{3}(\theta)+8 I_{41}(\theta) \cos ^{2}(\theta / 2) \\
& I_{41}(\theta)=3\left\{-5 H_{33}(\theta)+10 H_{23}(\theta)-5 H_{13}(\theta)+30 H_{32}(\theta)+26 H_{22}(\theta)-5 H_{31}(\theta)\right\} \cos ^{4} \omega_{0} \\
& +\left[6\left\{5 H_{33}(\theta)-10 H_{23}(\theta)+5 H_{13}(\theta)+15 H_{32}(\theta)-8 H_{22}(\theta)-3 H_{12}(\theta)+3 H_{21}(\theta)\right\}\right. \\
& \left.+12 P\left\{H_{22}(\theta)-H_{12}(\theta)+H_{21}(\theta)\right\}\right] \cos ^{2} \omega_{0}+3\left\{-5 H_{33}(\theta)+10 H_{23}(\theta)-5 H_{13}(\theta)\right. \\
& \left.-10 H_{22}(\theta)+6 H_{12}(\theta)-H_{11}(\theta)\right\}+4 P\left\{-3 H_{22}(\theta)+3 H_{12}(\theta)-H_{11}(\theta)\right\} \\
& I_{5}(\theta)=3 I_{3}(\theta)+8 I_{41}(\theta) \cos ^{2}(\theta / 2)+I_{51}(\theta) \\
& I_{51}(\theta)=\left[24\left\{H_{33}(\theta)-2 H_{23}(\theta)+H_{13}(\theta)+6 H_{32}(\theta)-6 H_{22}(\theta)+H_{31}(\theta)\right\} \cos ^{4} \omega_{0}\right. \\
& +48\left\{-H_{33}(\theta)+2 H_{23}(\theta)+H_{13}(\theta)-3 H_{23}(\theta)+2 H_{22}(\theta)+H_{12}(\theta)-H_{21}(\theta)\right\} \\
& \left.+64 P\left\{-H_{22}(\theta)+H_{12}(\theta)-H_{21}(\theta)\right\}\right] \cos ^{2} \omega_{0}+24\left\{H_{33}(\theta)-2 H_{23}(\theta)+H_{13}(\theta)\right. \\
& \left.+2 H_{22}(\theta)-2 H_{12}(\theta)+H_{11}(\theta)\right\}+64 P\left\{H_{22}(\theta)-H_{12}(\theta)+H_{11}(\theta)\right\}+64 P^{2} H_{11}(\theta)
\end{aligned}
$$

The terms $H_{i j}(\theta)$ are defined by 


$$
\begin{aligned}
H_{i j}(\theta)= & \frac{1}{\pi} \int_{\alpha=0}^{\pi} \int_{\gamma=0}^{\pi / 2} \cos ^{2(i-1)} \alpha \cos ^{2(j-1)} \gamma \frac{\sin ^{2}\left[U_{1} \cos \alpha\right]}{\left[U_{1} \cos \alpha\right]^{2}} \\
& \times \frac{\sin ^{2}\left[U_{\mathrm{d}} \sin \alpha \cos \gamma\right]}{\left[U_{\mathrm{d}} \sin \alpha \cos \gamma\right]^{2}} \frac{\sin ^{2}\left[U_{\mathrm{g}} \sin \alpha \sin \gamma\right]}{\left[U_{\mathrm{g}} \sin \alpha \sin \gamma\right]^{2}} \sin \alpha \mathrm{d} \alpha \mathrm{d} \gamma
\end{aligned}
$$

and the quantity $p$ is defined by $p=\left(\alpha_{\perp}-\alpha_{\mathrm{s}}\right) /\left(\alpha_{\|}-\alpha_{\perp}\right)=b_{\mathrm{t}} / \delta$.

In the case of the homogeneous one-dimensional rods with infinitesimally small values of $D$ and $G$, the terms $H_{i j}(\theta)$ reduce to

$$
H_{i}(\theta)=\frac{1}{2} \int_{0}^{\pi} \cos ^{2(i-1)} \alpha \frac{\sin ^{2}\left[U_{1} \cos \alpha\right]}{\left[U_{1} \cos \alpha\right]^{2}} \sin \alpha \mathrm{d} \alpha
$$

the integrations of which can be solved analytically as shown in the previous paper ${ }^{2}$, and the $H_{\nabla}$ scattered intensity reduces to

$$
\begin{aligned}
I_{H_{\mathrm{v}}}(\theta, \mu)= & K_{3}\left\{( 1 / 8 ) P _ { 4 } ( \operatorname { c o s } \omega _ { 0 } ) [ 1 0 5 \operatorname { c o s } ^ { 4 } ( \theta / 2 ) \operatorname { s i n } ^ { 2 } 2 \mu - 6 0 \operatorname { c o s } ^ { 2 } ( \theta / 2 ) + 1 2 ] \left[35 H_{3}(\theta)\right.\right. \\
& \left.\left.-30 H_{2}(\theta)+3 H_{1}(\theta)\right]+10 P_{2}\left(\cos \omega_{0}\right)\left[3 \cos ^{2}(\theta / 2)-2\right]\left[3 H_{2}(\theta)-H_{1}(\theta)\right]+28 H_{1}(\theta)\right\}
\end{aligned}
$$

where $P_{2}(x)=\left(3 x^{2}-1\right) / 2$, and $P_{4}(x)=\left(35 x^{4}-30 x^{2}+3\right) / 8$.

The angular dependences of the $H_{\nabla}$ scattering intensities from the system with respect to $\mu$ depend upon the relative values of $I_{1}$ and $I_{2}$ for a given $\theta$ as seen in eq 14. For instance, when the absolute values of $I_{1}(\theta)$ are greater than those of $I_{2}(\theta)$, the scattering patterns have large $\mu$-dependences. The scattering patterns are of the $x$-type (with maximum and minimum intensities at odd and even multiples of $\mu=45^{\circ}$, respectively) or of the +-type (with maximum and minimum intensities at even and odd multiples of $\mu=45^{\circ}$, respectively) appearances depending on the sign of $I_{1}(\theta)$; i.e., the $\times$-type pattern for positive $I_{1}(\theta)$ and the +-type for negative $I_{1}(\theta)$.

On the other hand, if the absolute values of $I_{1}(\theta)$ are smaller than those of $I_{2}(\theta)$, then the scattering patterns have little $\mu$-dependences and show the circular-type appearance. The critical conditions at which the scattering patterns change their appearance from the $\times$-type to circular type, from the circular to the +-type, and etc., depend upon the size and shape of the plate as well as the orientation of the optical axes $\omega_{0}$ within the plate and the scattering angle $\theta$, as seen in eq 16 to 18 . Therefore the shape of the scattering patterns generally depend upon $\theta$ in a manner determined by the value of $\omega_{0}$, and the shape and size of the plate. For example, the scattered pattern may change from the +-type to the circular-type and to the $\times$-type with increasing $\theta$ for a given value of $\omega_{0}\left(=45^{\circ}\right)$ as shown in later in Figure 4.

In contrast to the scattering from the plates, the $H_{\nabla}$ scattering from the one-dimensional rods is given in a form of

$$
I_{H_{\mathrm{V}}}(\theta, \mu)=K_{4}\left[\sin ^{2} 2 \mu P_{4}\left(\cos \omega_{0}\right) F_{1}\left(U_{1}\right)+F_{2}\left(U_{1}\right)\right]
$$

where $F_{1}$ is a function of $U_{1}$, and $F_{2}$ depends also upon $P_{4}\left(\cos \omega_{0}\right)$ and $P_{2}\left(\cos \omega_{0}\right)$ as seen in eq 23 and 24. The functions $F_{1}$ and $F_{2}$ are positive irrespective of the values $U_{1}$, i.e., $\theta$ and $L$, so that the shape of the scattering patterns are the $\times$-type, circular-type, and +-type irrespective of values of $\theta$ and $L$ for $\omega_{0}$ satisfying $P_{4}\left(\cos \omega_{0}\right)$ to be positive, zero and negative, respectively, for the one-dimensional rods.

\section{Results of Numerical Calculations}

Numerical calculations were carried out for the plates with same volume but different shapes; (i) the plate with $L / \lambda=30, D / L=0.2$ and $G / L=$ 0.067 , and (ii) the plate with $L / \lambda=30, D / L=$ 0.067 and $G / L=0.2$. The length $D$ is the lateral width of the plane containing the optical axes $\boldsymbol{d}$ and the vector $\boldsymbol{l}$ as shown in Figure 2 .

In Figures 3 and 4 are shown the calculated $H_{\nabla}$ scattering patterns from the assembly of the plate with the length $L / \lambda=30$, the width $D / L=$ 0.067 and the thickness $G / L=0.2$, and with $L / \lambda=30, D / L=0.2$ and $G / L=0.067$, respectively. The $H_{\mathrm{v}}$ scattering patterns from the plate with a thin width $D$ (Figure 3) are similar to those from the one-dimensional rods. The scattering patterns change from the $\times$-type to the nearly circular-type, the +-type, the nearly circular-type 


\section{Hv patterns $L / \lambda=30, q=0.067, s=0.2$}
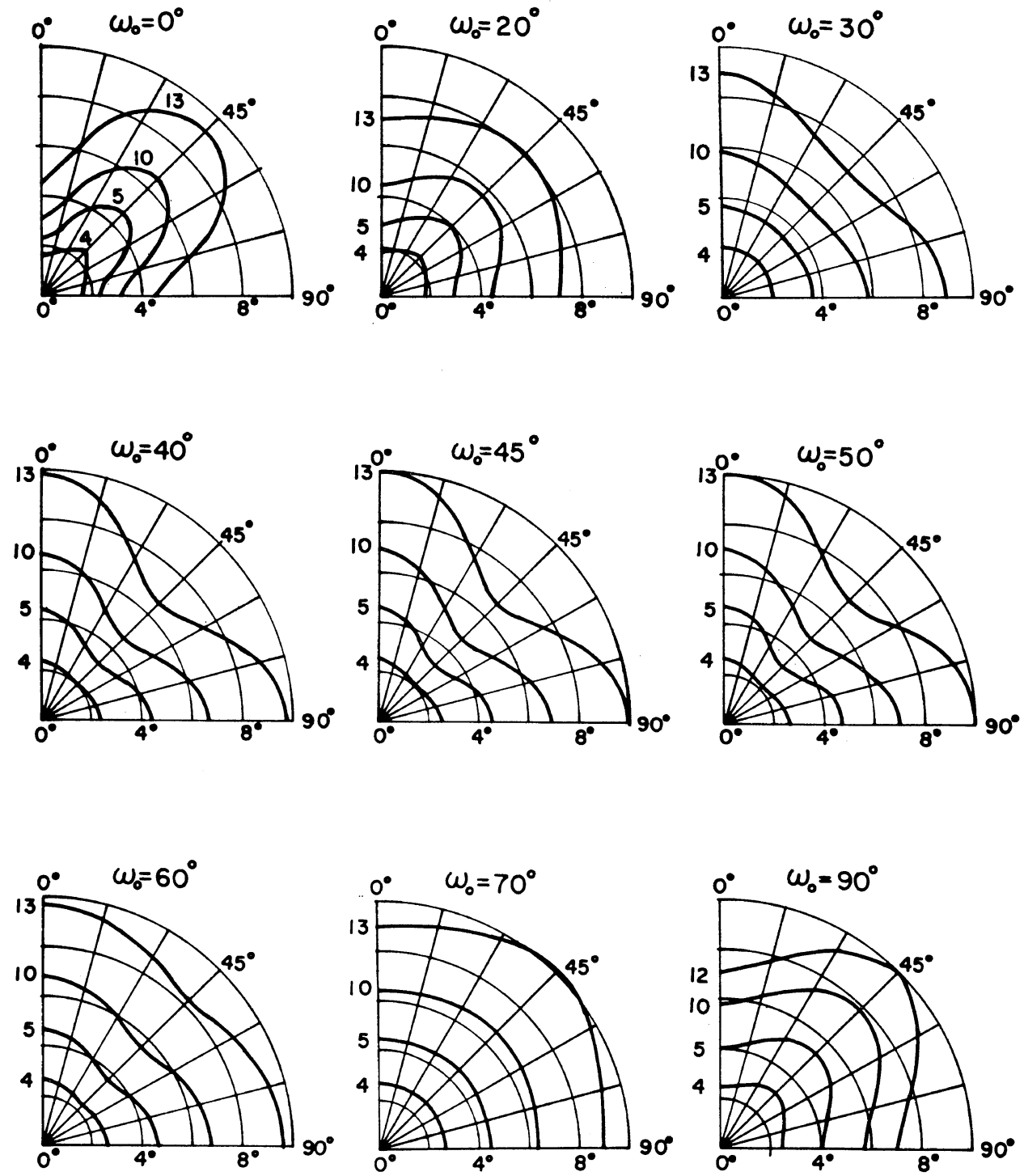

Figure 3. $H_{\mathrm{v}}$ scattering patterns from the random assembly of the anisotropic plates with $L / \lambda=30$, $D / L=0.067$, and $G / L=0.2$ in three-dimensional space.

and to the $\times$-type with increasing value of $\omega_{0}$. The critical values of $\omega_{0}$ at which the patterns change their appearances, however, depend upon the size and shape of the plate in contrast to the one-dimensional rod. The angular dependences of the $H_{\mathrm{v}}$ scattering intensities with respect to the azimuthal angle $\mu$ increase with increasing scattering angle as in the case of the one-dimensional rod except for the case of the value of $\omega_{0}$ being nearly equal to $20^{\circ}$ where the effect of finite lateral width still remains.

The effects of increasing the width $D / L$ on the $H_{\checkmark}$ scattering patterns are clearly seen in Figure 4. It is seen, by comparing Figures 3 


\section{Hv patterns $L / \lambda=30, q=0.2, s=0.067$}
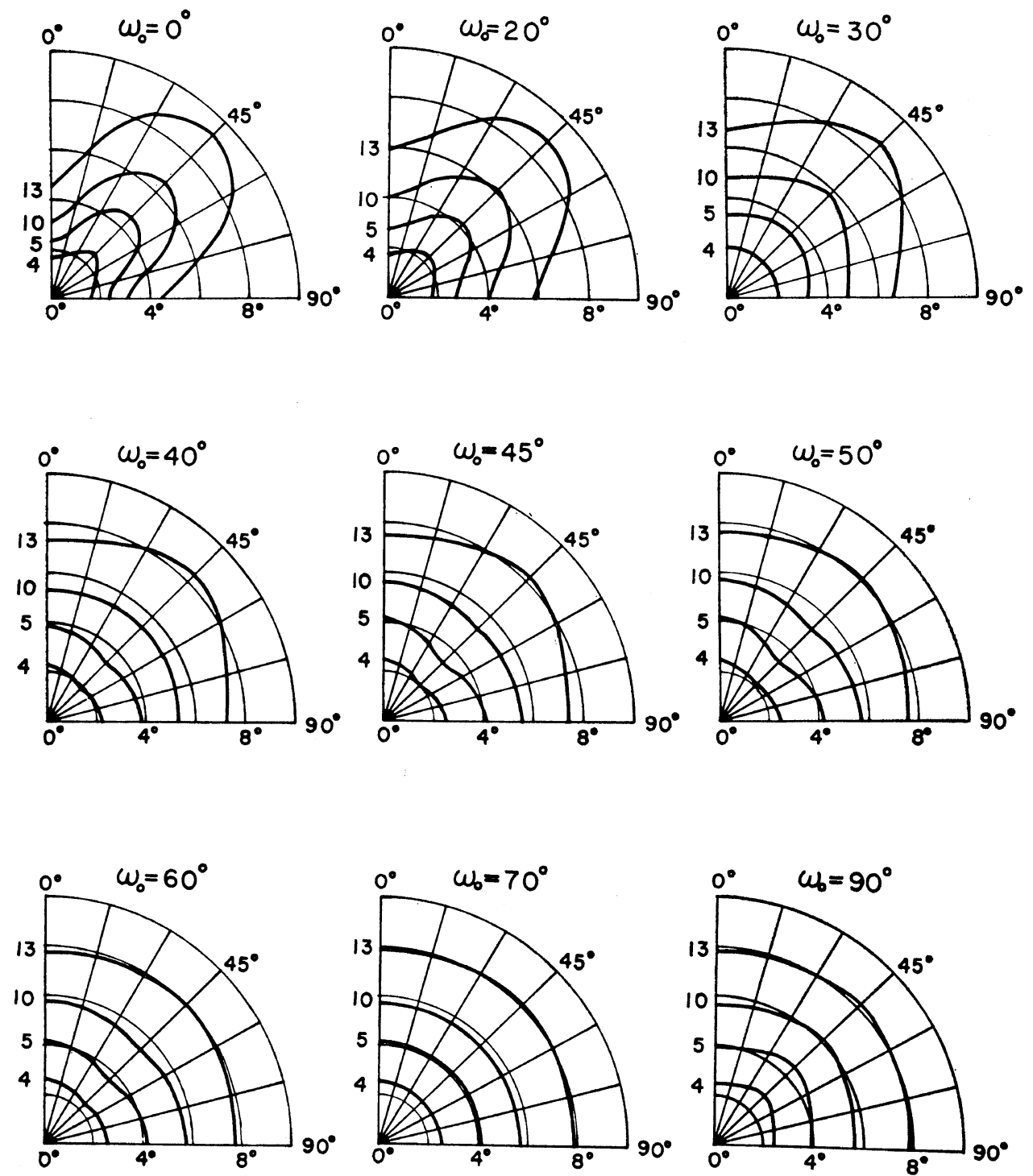

Figure 4. $H_{\mathrm{v}}$ scattering patterns from the random assembly of the plates with $L / \lambda=30, D / L=0.2$, and $G / L=0.067$ in three-dimensional space.

and 4 , that the critical values of $\omega_{0}$ at which the scattering patterns change their appearance are quite different from the previous case. The $\mu$-dependence of the scattered intensities are complex and does not continuously increase with increasing $\theta$ as in the plate with thin width $D$. For the +-type (e.g., the patterns for $\omega_{0}=40$

to $\left.60^{\circ}\right)$, the $\mu$-dependence increases and then decreases to a minimum, and again increases with increasing $\theta$ so that the scattering patterns are the +-type at small $\theta$ and the $\times$-type at large $\theta$. Similar tendencies are also expected for the scattering from the cylindrical particles studied by Hayashi and Kawai, although they 


\section{Vv patterns}

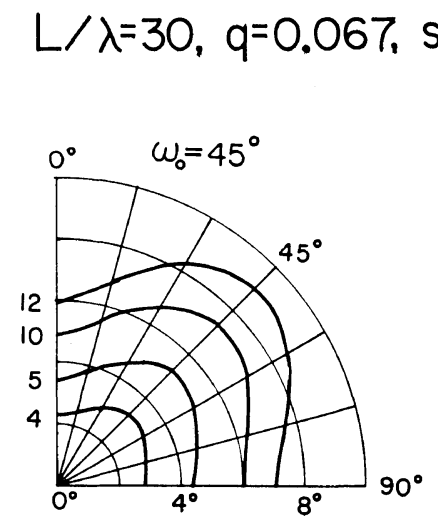

$s=0.2, p=-1 / 3$
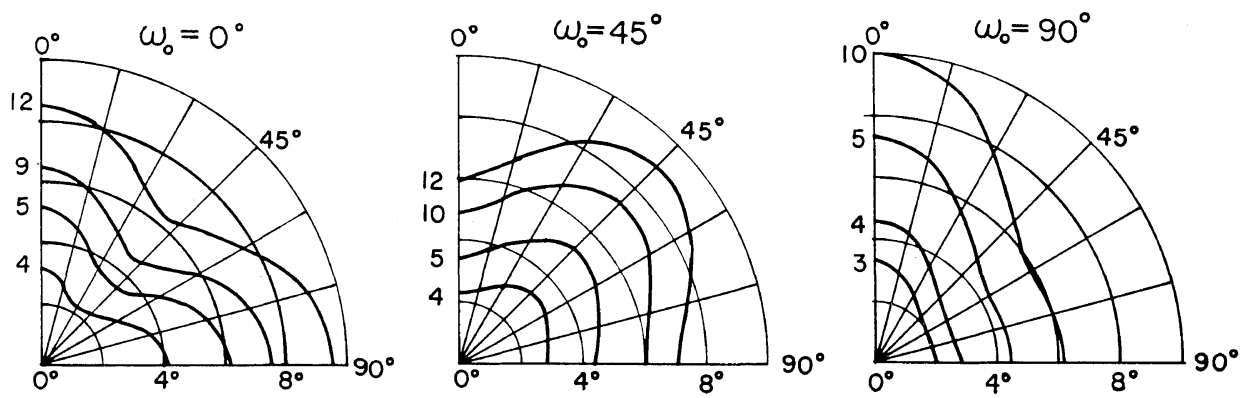

Figure 5. $V_{\mathrm{V}}$ scattering patterns from the random assembly of the plates with $L / \lambda=30, D / L=0.067$, and $G / L=0.2$ in three-dimensional space; $p=-1 / 3$.

\section{Vv patterns $L / \lambda=30, q=0.2, s=0.067, p=-1 / 3$}
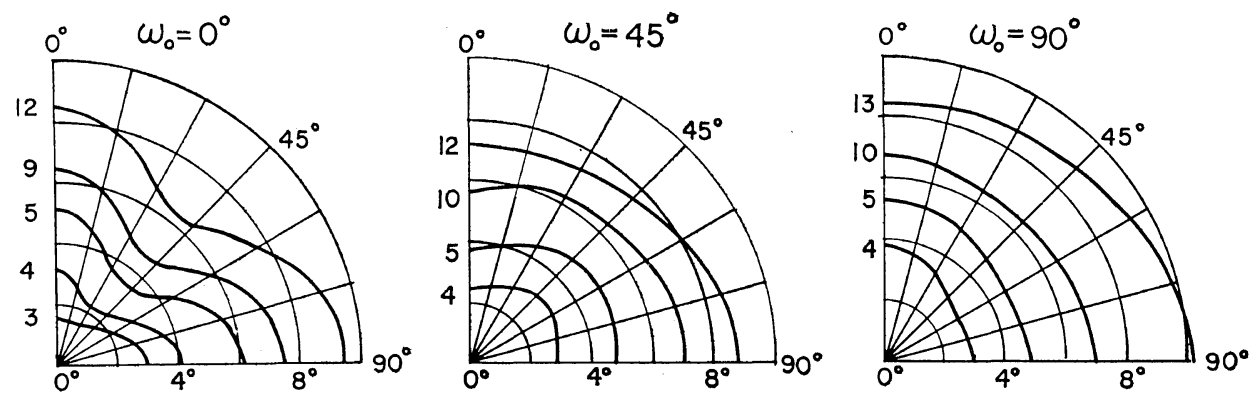

Figure 6. $V_{\mathrm{v}}$ scattering patterns from the random assembly of the plates with $L / \lambda=30, D / L=0.2$, and $G / L=0.067$ in three-dimensional space; $p=-1 / 3$.

did not show up clearly. ${ }^{3}$ The tendencies may be attributed to the effect of finite lateral dimensions of the rod-like texture.

As seen in eq 15, the $V_{\mathrm{v}}$ scattering depends upon the density fluctuations related to the quantity $p$ as well as the orientation and anisotropy fluctuations related to the quantities $\omega_{0}$ and $\delta_{0}$. The term $I_{3}(\theta)$ depends only upon the anisotropy and orientation fluctuations, while the terms $I_{4}(\theta)$ and $I_{5}(\theta)$ depend also upon the density fluctuations.

The calculated $V_{\mathrm{v}}$ scattering patterns are shown in Figures 5 and 6 for $p=-1 / 3$, the value of which gives the predominant contribution of the anisotropy and orientation fluctuations compared with the contribution of the density fluctuation. If the term $I_{3}(\theta)$ predominates over the others, the $V_{v}$ patterns become four- fold symmetric with respect to $\mu$ as seen in the pattern close to that for $\omega_{0}=45^{\circ}$ in Figure 5. If the term $I_{4}(\theta)$ is predominant, the patterns become two-fold symmetric as seen in the figures. The density fluctuation increases the contribution of the term $I_{5}(\theta)$, so that the scattering patterns become the circular-type if the absolute value of $p$ is large. It is seen, by comparing Figures 5 and 6 , that the relative contribution of the terms depends again upon the value of $\omega_{0}$ and the size and shape of the plate as well as the value of $p$, and that the $V_{\mathrm{v}}$ patterns from the plate with thin width (Figure 5) are similar to those from the one-dimensional rod.

\section{RANDOM ASSEMBLY OF THE ANISOTROPIC PLATES IN TWO-DIMENSIONAL SPACE}

The two-dimensional distribution of the plates 
Light Scattering from Plate-like Crystalline Textures

is given by setting $\Phi=0$ in Figure 1 . Two cases are considered for orientation of the plates with respect to $\phi$; (i) the case of $\psi=0^{\circ}$ where the optic axes are constrained in the plane parallel to $O Y Z$, and (ii) the case of $\phi=90^{\circ}$ where the plane composed of the vectors $\boldsymbol{l}$ and $\boldsymbol{m}$ is oriented perpendicular to the plane of $O Y Z$.

Case of $\phi=0^{\circ}$

The calculations can be performed in a manner similar to the previous calculations based upon the coordinate system as shown in Figure 1. The results for $\phi=0^{\circ}$ are given by,

$$
\begin{aligned}
I_{H_{\mathrm{V}}}(\theta, \mu)= & K_{5}\left\{F _ { 1 } ( \operatorname { c o s } \omega _ { 0 } ) \left[8 G_{3}(\theta)-8 G_{2}(\theta)\right.\right. \\
& \left.+G_{1}(\theta)\right] \sin ^{2} 2 \mu+4 F_{1}\left(\cos \omega_{0}\right)\left[G_{2}(\theta)\right. \\
& \left.\left.-G_{3}(\theta)\right]+4 F_{2}\left(\cos \omega_{0}\right) G_{1}(\theta)\right\}
\end{aligned}
$$

and

$$
\begin{aligned}
I_{V_{\nabla}}(\theta, \mu)= & K_{6}\left\{F _ { 1 } ( \operatorname { c o s } \omega _ { 0 } ) \left[8 G_{3}(\theta)-8 G_{2}(\theta)\right.\right. \\
& \left.+G_{1}(\theta)\right] \cos 4 \mu \\
& +4(2 p+1) F_{3}\left(\cos \omega_{0}\right)\left[2 G_{2}(\theta)\right. \\
& \left.-G_{1}(\theta)\right] \cos 2 \mu+[8 p(p+1) \\
& \left.\left.+24 F_{2}\left(\cos \omega_{0}\right)+3 F_{1}\left(\cos \omega_{0}\right)\right] G_{1}(\theta)\right\}
\end{aligned}
$$

where the functions $G_{i}(\theta)$ and $F_{i}(x)$ are given by

$$
\begin{aligned}
G_{i}(\theta)= & \frac{2}{\pi} \frac{\sin ^{2}\left[w_{\mathrm{g}} \tan (\theta / 2)\right]}{\left[w_{\mathrm{g}} \tan (\theta / 2)\right]^{2}} \\
& \times \int_{0}^{\pi / 2} \cos ^{2(i-1)} t \frac{\sin ^{2}\left[w_{1} \cos t\right]}{\left[w_{1} \cos t\right]^{2}} \\
& \times \frac{\sin ^{2}\left[w_{\mathrm{d}} \sin t\right]}{\left[w_{\mathrm{d}} \sin t\right]^{2}} \mathrm{~d} t
\end{aligned}
$$

$$
\begin{aligned}
& W_{1}=\pi(L / \lambda) \sin \theta, \quad W_{\mathrm{d}}=\pi(D / \lambda) \sin \theta, \\
& W_{\mathrm{g}}=\pi(\boldsymbol{G} / \lambda) \sin \theta
\end{aligned}
$$

and

$$
\begin{aligned}
& F_{1}(x)=8 x^{4}-8 x^{2}+1, \quad F_{2}(x)=x^{2}-x^{4}, \\
& F_{3}(x)=2 x^{2}-1
\end{aligned}
$$

It is seen, from eq 27, that the thickness $G$ along the propagation direction of the incident beam affects only the absolute intensities of $H_{\mathrm{v}}$ and $V_{\mathrm{v}}$ scatterings through the term $\left(\sin ^{2}\left[w_{\mathrm{g}} \tan (\theta / 2)\right] /\left[w_{\mathrm{g}} \tan (\theta / 2)\right]^{2}\right)$, the value of which is close to unity at very small scattering angles. When $D=G=0$, the eq 25 and 26 reduce to the case calculated by Stein, et al, for the two-dimensional distribution of the one-dimensional rods, the analytical results of which were given by Hashimoto, et al. ${ }^{6}$.

As seen in eq 25 , the $H_{\mathrm{v}}$ scattering from the plates is independent of $\mu$ irrespective of the size and shape when the value of $\omega_{0}$ satisfies the condition, $F_{1}\left(\cos \omega_{0}\right)=0$. Therefore the scattering pattern becomes the circular type for $\omega_{0}=$ $22.5^{\circ}$ and $67.5^{\circ}$ irrespective of $\theta$ both for the one-dimensional rods and for the three-dimensional plates.

Similarly to the $H_{\mathrm{v}}$ scattering from the onedimensional rods, the scattering from the plates with dimensions of $L / \lambda=30, D / L=0.2, G / L=$ 0.067 , and $L / \lambda=30, D / L=0.067$ and $G / L=0.2$ is the $\times$-type, the circular-type, and the +-type when $F_{1}\left(\cos \omega_{0}\right)$ is positive (i.e., for $\omega_{0}$ satisfying $0^{\circ} \leq \omega_{0}<22.5^{\circ}$ and $67.5^{\circ}<\omega_{0} \leq 90^{\circ}$ ), zero

\section{Hv patterns $L / \lambda=30, q=0.067, s=0.2$}
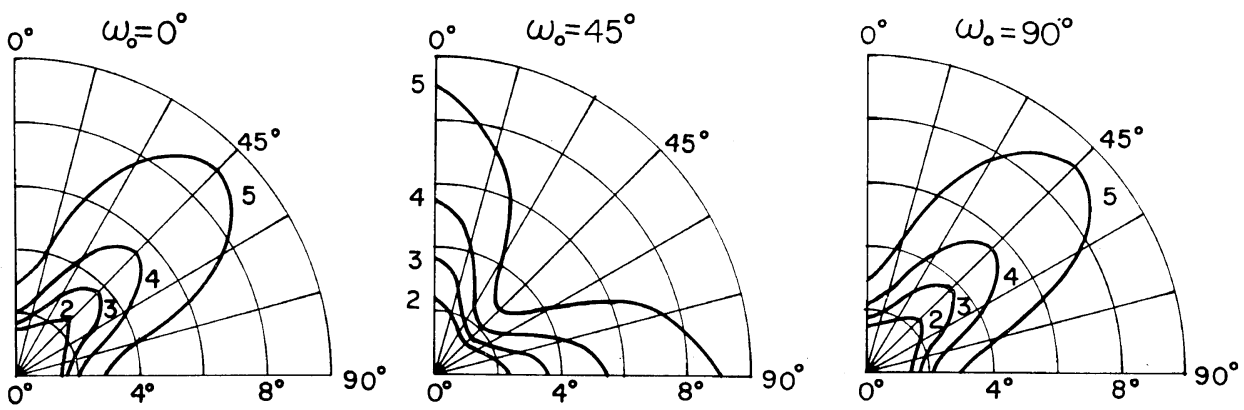

Figure 7. $H_{\mathrm{V}}$ scattering patterns from the random assembly of the plates with $L / \lambda=30, D / L=0.067$, and $G / L=0.2$ in two-dimensional space; $\phi=0^{\circ}$. 


\section{$H v$ patterns $L / \lambda=30, q=0.2, s=0.067$}
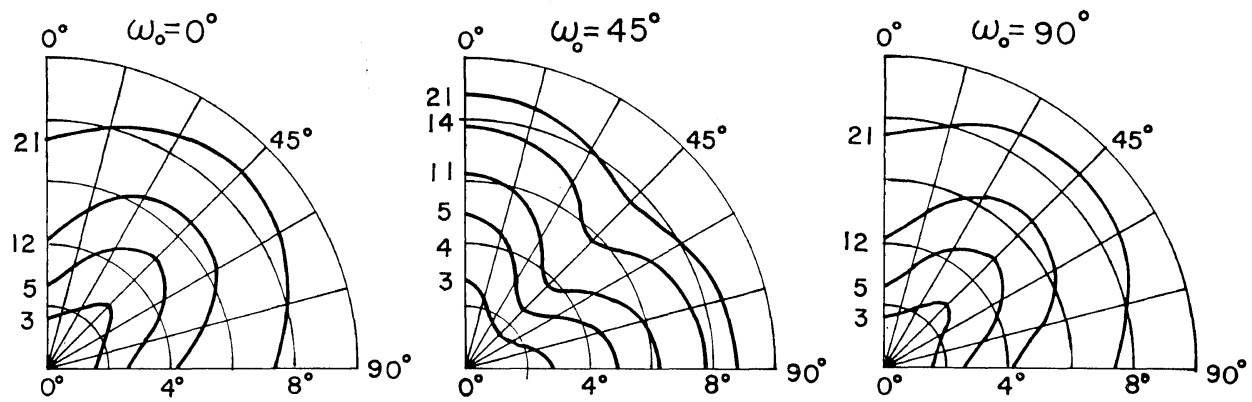

Figure 8. $H_{\mathrm{v}}$ scattering patterns from the random assembly of the plates with $L / \lambda=30, D / L=0.2$, and $G / L=0.067$ in two-dimensional space; $\phi=0^{\circ}$.

(i.e., for $\omega_{0}$ satisfying $\omega_{0}=22.5^{\circ}$ and $67.5^{\circ}$ ) and negative (i.e., for $\omega_{0}$ satisfying $22.5^{\circ}<\omega_{0}<67.5^{\circ}$ ), respectively. This is because the term $\left(8 G_{3}-\right.$ $\left.8 G_{2}+G_{1}\right)$ is positive for any values of $\theta$ for the one dimensional rods and for the range of $\theta$ examined for the plates.

\section{Numerical Calculations}

In Figures 7 and 8 are shown the results of calculated $H_{\mathrm{v}}$ scattering patterns for the plates. As seen in eq 25 the patterns for $\omega_{0}=0$ and $90^{\circ}$ are identical in contrast to the previous case of the three-dimensional distribution of the plates. The $\mu$-dependence of the $H_{\mathrm{v}}$ scattering from the plate with thin lateral dimension $D$ increases with increasing scattering angles as seen in Figure 7. The tendency is similar to that seen for the one-dimensional rods oriented randomly in a two-dimensional plane. ${ }^{1,6}$ For the plate having a larger lateral dimension $D$, the $\mu$-dependence of the $H_{\mathrm{v}}$ pattern increases and then decreases with increasing scattering angles as seen in Figure 8.

In Figures 9 and 10 are shown the calculated $V_{\mathrm{v}}$ scattering patterns for $p=-1 / 3$ where the anisotropy and orientation fluctuations contribute more than the density fluctuation. It is seen, by comparing the figures, that the $V_{\mathrm{v}}$ patterns for the plates with small $D$ is similar to those for the one-dimensional rods, and that upon increasing the thickness $D$, the $\mu$-dependence becomes smaller at high scattering angles.

When the value of $\omega_{0}$ is $45^{\circ}$, the term $F_{3}$ in

\section{Vv patterns $L / \lambda=30, q=0.067, s=0.2, p=-1 / 3$}
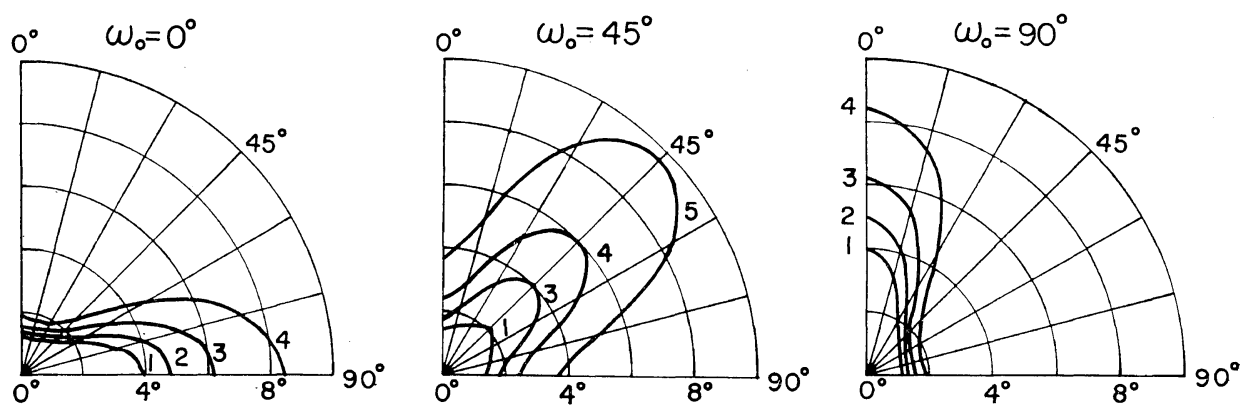

Figure 9. $V_{\mathrm{V}}$ scattering patterns from the random assembly of the plates with $L / \lambda=30, D / L=0.067$, and $G / L=0.2$ in two-dimensional space; $\phi=0^{\circ}$ and $p=-1 / 3$. 


\section{$V v$ patterns $L / \lambda=30, q=0.2, s=0.067, p=-1 / 3$}
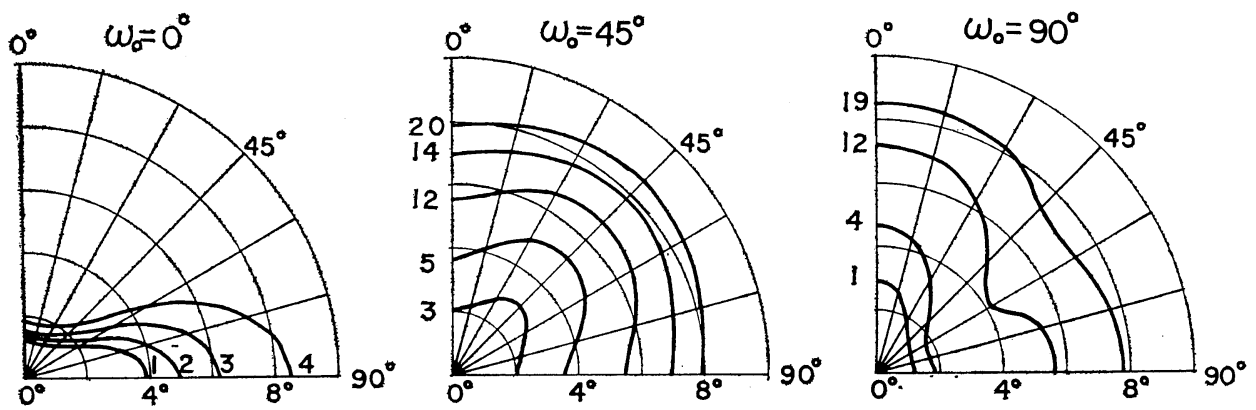

Figure 10. $V_{\mathrm{V}}$ scattering patterns from the random assembly of the plates with $L / \lambda=30, D / L=0.2$, and $G / L=0.067$ in two-dimensional space; $\phi=0^{\circ}$ and $p=-1 / 3$.

eq 26 becomes zero, so that the $V_{v}$ patterns become four-fold symmetric in the $\mu$-dependence. Simultaneously the coefficient of $\cos 4 \mu$ becomes negative, giving rise to the $\times$-type $V_{\checkmark}$ patterns as seen in the figures.

Case of $\phi=90^{\circ}$

The $H_{\mathrm{v}}$ and $V_{\mathrm{v}}$ scatterings can be calculated for the case of $\psi=90^{\circ}$ in a manner similar to the calculations, for $\phi=0^{\circ}$. The results are given by

$$
\begin{aligned}
I_{H_{\mathrm{V}}}(\theta, \mu)= & K_{7} \cos ^{4} \omega_{0}\left\{\left[8 E_{3}(\theta)-8 E_{2}(\theta)\right.\right. \\
& \left.\left.+E_{1}(\theta)\right] \sin ^{2} 2 \mu+4\left[E_{2}(\theta)-E_{3}(\theta)\right]\right\} \\
I_{V_{\mathrm{V}}}(\theta, \mu)= & K_{8}\left\{\operatorname { c o s } ^ { 4 } \omega _ { 0 } \left[8 E_{3}(\theta)-8 E_{2}(\theta)\right.\right. \\
& \left.+E_{1}(\theta)\right] \cos 4 \mu \\
& -4 \cos ^{2} \omega_{0}\left(2 p+\cos ^{2} \omega_{0}\right)\left[E_{1}(\theta)\right.
\end{aligned}
$$

$$
\begin{aligned}
& \left.-2 E_{2}(\theta)\right] \cos 2 \mu+\left[8 p^{2}+8 p \cos ^{2} \omega_{0}\right. \\
& \left.\left.+3 \cos ^{4} \omega_{0}\right] E_{1}(\theta)\right\}
\end{aligned}
$$

where $E_{i}(\theta)$ is given by

$$
\begin{aligned}
E_{i}(\theta)= & \frac{2}{\pi} \frac{\sin ^{2}\left[W_{\mathrm{d}} \tan (\theta / 2)\right]}{\left[W_{\mathrm{d}} \tan (\theta / 2)\right]^{2}} \\
& \times \int_{0}^{\pi / 2} \cos ^{2(i-1)} t \frac{\sin ^{2}\left[W_{1} \cos t\right]}{\left[W_{1} \cos t\right]^{2}} \\
& \times \frac{\sin ^{2}\left[W_{\mathrm{g}} \sin t\right]}{\left[W_{\mathrm{g}} \sin t\right]^{2}} \mathrm{~d} t
\end{aligned}
$$

In this case the term related to the width $D$ (i.e., the width of the sheet parallel to the propagation direction of the incident beam) affects the absolute intensities of the $H_{\mathrm{v}}$ and $V_{\mathrm{v}}$ scatterings. In contrast to the previous cases, the

\section{Hv patterns $L / \lambda=30, q=0.2, s=0.067$}
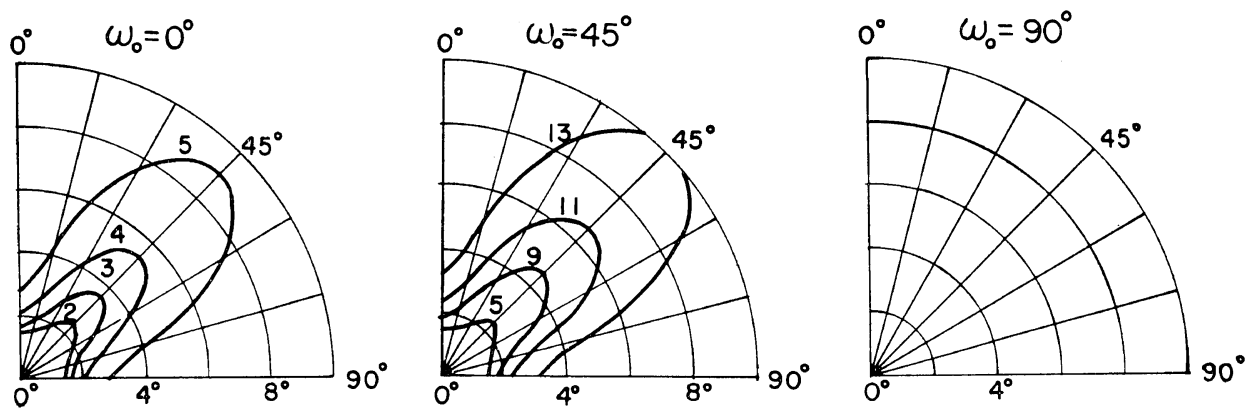

Figure 11. $H_{\mathrm{v}}$ scattering patterns from the random assembly of the plates with $L / \lambda=30, D / L=0.2$, and $G / L=0.067$ in two-dimensional space; $\phi=90^{\circ}$. 


\section{$V v$ patterns $L / \lambda=30, q=0.2, s=0.067, p=-1 / 3$}
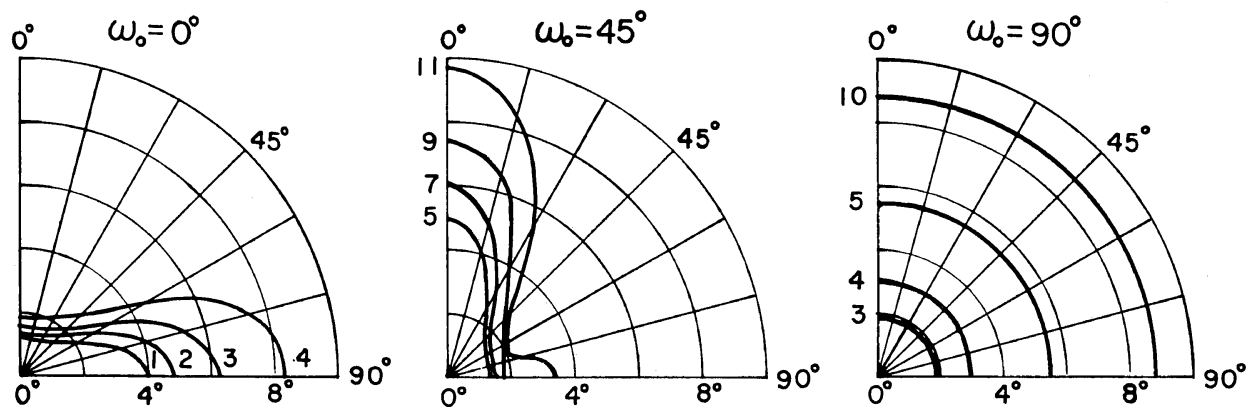

Figure 12. $V_{\mathrm{v}}$ scattering patterns from the random assembly of the plates with $L / \lambda=30, D / L=0.2$, and $G / L=0.067$ in two-dimensional space; $\phi=90^{\circ}$ and $p=-1 / 3$.

value of $\omega_{0}$ does not affect the relative intensity distributions of the $H_{\mathrm{v}}$ scattering, but affects only the absolute intensities as seen in eq 29. In case where $\omega_{0}=90^{\circ}$, the orientation contribution to the $H_{\mathrm{v}}$ and $V_{\mathrm{v}}$ scatterings becomes zero, so that the $H_{\mathrm{v}}$ scattering intensities become zero, and the $V_{\mathrm{v}}$ scattering arises only from the density term, i.e., the term $p^{2} E_{1}(\theta)$.

\section{Numerical Calculations}

In Figures 11 and 12 are shown the calculated $H_{\mathrm{v}}$ and $V_{\mathrm{v}}$ scattering patterns for the plates with $L / \lambda=30, D / L=0.2$ and $G / L=0.067$, respectively. The $H_{\mathrm{v}}$ scattering patterns are the $\times$-type irrespective of the values of $\omega_{0}$ except for the case of $\omega_{0}=90^{\circ}$ where the $H_{\mathrm{v}}$ scattering intensity becomes zero. This is interpreted as arising

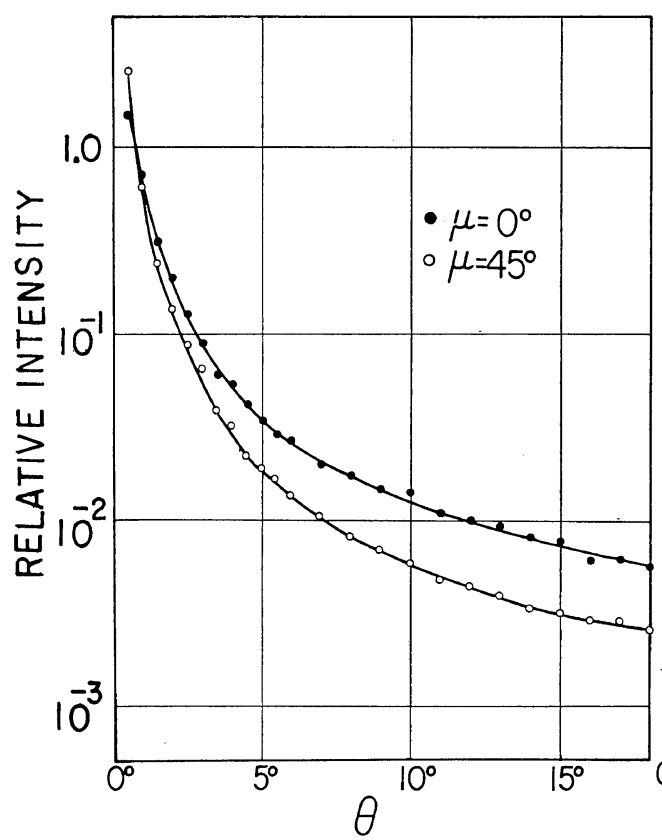

(a)

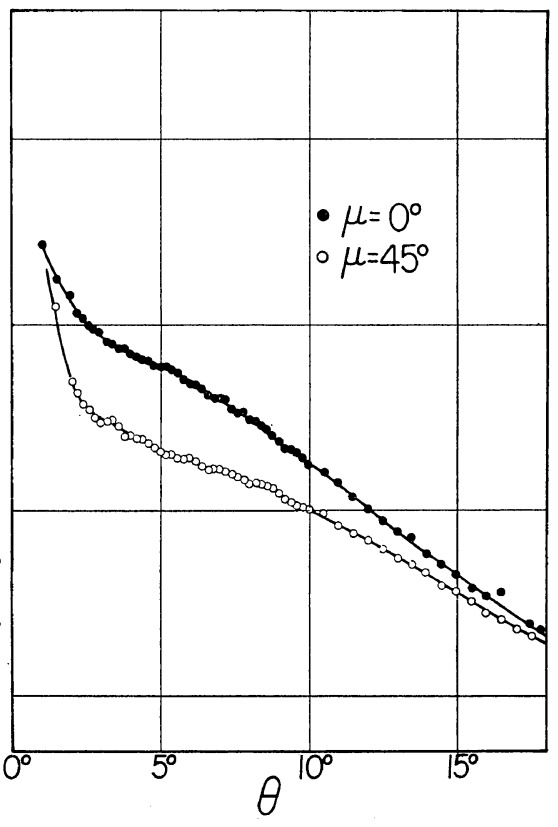

(b)

Figure 13. The experimental $H_{\nabla}$ scattered intensity distributions at $\mu=0$ and $45^{\circ}$ for two types of the denatured collagen films. The intensities were photometrically measured. 
from the fact that the term related to $\sin ^{2} 2 \mu$ in eq 29 is positive. The $V_{\nabla}$ pattern is the circular type for $\omega_{0}=90^{\circ}$, because the scattering arises purely from the density contribution as discussed above. The $H_{\nabla}$ and $V_{\nabla}$ scattering patterns for the plates having $L / \lambda=30, D / L=0.067$ and $G / L=$ 0.2 are similar to those discussed above except for the fact that the $\mu$-dependence does not increase continuously, but increases and then decreases with increasing scattering angles, this tendency being in agreement with that of the previous cases.

\section{DISCUSSIONS}

In Figures $13 a$ and $13 b$ are shown the experimental $H_{\mathrm{v}}$ scattered intensity distributions at $\mu=0$ and $45^{\circ}$ for two types of denatured collagen films. ${ }^{6}$ In the previous paper, ${ }^{6}$ the scattering was shown to be rod-like, and the texture of the collagen films was suggested to be oriented randomly in a plane parallel to the film surfaces.
In Figures $14 \mathrm{a}$ and $14 \mathrm{~b}$ are shown the calculated $H_{v}$ scattered intensity distributions at various value of $\mu$ for the two-dimensional distribution of the plates under $\phi=0^{\circ}$. The plates are assumed to have a value of $\omega_{0}$ equal to $45^{\circ}$.

By comparing Figures 13 and 14, the tendency shown in Figure 13a that the $\mu$-dependence of the $H_{\mathrm{v}}$ scattering increases with increasing $\theta$ is shown to be explained by considering the rodlike texture having small lateral dimension $D$, while the tendency shown in Figure 13b that the $\mu$-dependence decreases with increasing $\theta$ is shown to be due to the texture having a larger lateral dimension $D$. In the previous paper, ${ }^{6}$ it was shown that the internal disorders of the rod-like texture also account for the latter tendency. It does not seem probable however that these disorders alone, account for the large decrease of the $\mu$-dependence at larger angles as shown in Figure 13b, unless very large disorders are involved in the texture.

It should be noted that the theoretical intensity

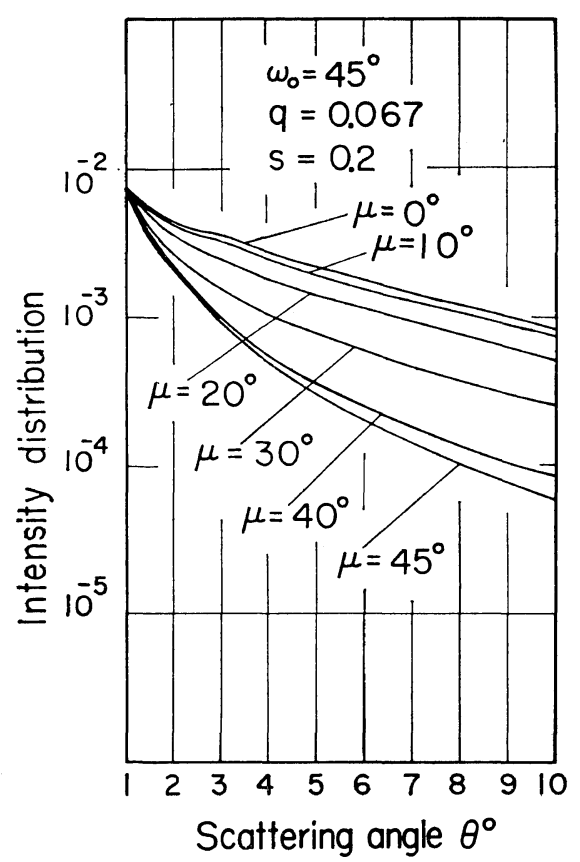

(a)

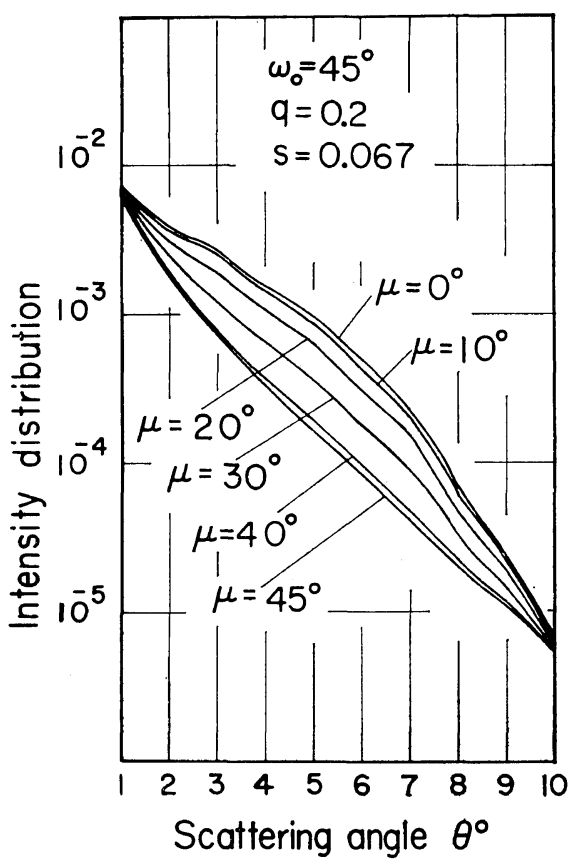

(b)

Figure 14. The calculated $H_{\mathrm{v}}$ scattered intensity distributions for the random assembly of the plates, (a) $L / \lambda=30, D / L=0.067$, and $G / L=0.2$, and (b) $L / \lambda=30, D / L=0.2$, and $G / L=0.067$ in twodimensional space under $\phi=0^{\circ}$. The value of $\omega_{0}$ is assumed to be $45^{\circ}$. 
distributions (Figure 14) deviate from the experimental intensity distributions (Figure 13) at very small angles. The deviation can be attributed to an interparticle interference effect which was neglected in the theoretical calculations.

\section{REFERENCES}

1. M. B. Rhodes and R. S. Stein, J. Polym. Sci., Part A-2, 7, 1539 (1969).

2. M. Moritani, N. Hayashi, A. Utsuo, and $\mathbf{H}$. Kawai, Polymer J. 2, 74 (1971).

3. N. Hayashi and H. Kawai, ibid., 3, 140 (1972).

4. J. J. van Aartsen, Eur. Polym. J., 6, 1095 (1970).

5. Y. Murakami, N. Hayashi, T. Hashimoto, and H. Kawai, Polymer J., 4, 452 (1973).

6. T. Hashimoto, Y. Murakami, N. Hayashi, and H. Kawai, ibid., 6, 132 (1974).

7. M. B. Rhodes and R.S. Stein, J. Appl. Phys., 39, 4093 (1968).
8. N. Hayashi, Y. Murakami, M. Moritani, T. Hashimoto, and H. Kawai, Polymer J., 4, 560 (1973).

9. T. Hashimoto, Y. Murakami, Y. Okamori, and H. Kawai, submitted to Polymer $J$.

10. G. C. Adams and R. S. Stein, J. Polym. Sci., Part A-2, 6, 31 (1968).

11. J.C.W. Chien and E.P. Chang, Macromolecules, 5, 610 (1972).

12. J. Borch and R. H. Marchessault, J. Polym. Sci., Part C, 28, 153 (1969).

13. R. J. Samuels, ibid. Part A-2, 7, 1197 (1969).

14. See for example, H.C. van de Hulst, "Light Scattering by Small Particles", John Wiley and Sons Inc., New York, N. Y., 1957.

15. A. E. Keijzers, J. J. van Aartsen, and W. Prins, J. Amer. Chem. Soc., 90, 3167 (1968).

16. S. B. Clough, J. J. van Aartsen, and R. S. Stein, J. Appl. Phys., 36, 3072 (1965). 